\title{
Nonlinear free-carrier velocity induced by intense Terahertz pulse in
}

\section{photoexcited semiconductor materials}

\author{
F. H. Su ${ }^{(1) *}$, G. Sharma ${ }^{(2)}$, F. Blanchard ${ }^{(2)}$, L. Razzari ${ }^{(2,3)}$, A. Ayesheshim ${ }^{(4)}$, R. Morandotti ${ }^{(2)}$, T. \\ Ozaki $^{(2)}$, and F. A. Hegmann ${ }^{(4)}$ \\ (1) Key Laboratory of Materials Physics, Institute of Solid State Physics, Chinese Academy of \\ Sciences, Hefei 230031, China \\ (2) INRS-EMT, Advanced Laser Light Source, Université du Québec, Varennes, Québec J3X 1S2, \\ Canada \\ ${ }^{(3)}$ Dipartimento di Elettronica, Università di Pavia, via Ferrata 1, 27100 Pavia, Italy \\ ${ }^{(4)}$ Department of Physics, University of Alberta, Edmonton, Alberta T6G 2G7, Canada
}

\begin{abstract}
:
The transient absorption bleaching and velocity overshoot of photoexcited carriers in GaAs and Si have been observed by the intense few-circle terahertz (THz) probe pulse in the optical pump-terahertz probe (OPTP) configuration. The free-carrier $\mathrm{THz}$ nonlinearity is attributed to the transient electron redistribution in conduction band induced by the strong THz electric field component.
\end{abstract}

Key words: Terahertz, Absorption bleaching, Velocity overshoot, Nonlinearity, GaAs, Si, Photoexcited, THz

\section{INTRODUCTION}

The developments of intense coherent $\mathrm{THz}$ source stimulated the explorations on the nonlinear effects of materials in $\mathrm{THz}$ frequency range in recent years ${ }^{[1-14]}$. The intense-THz induced exciting phenomena include dynamical Franz-Keldysh effect (DFKE) $[5,6]$, cross-phase modulation ${ }^{[7]}$, ultrafast impact ionization ${ }^{[8,9]}$, photoluminescence quenching ${ }^{[10]}$ etc. have been reported. It has been found that the intense $\mathrm{THz}$ electric field can produce an additional long-lived coherent $\mathrm{THz}$ emission ${ }^{[11]}$, and induce the carrier-wave Rabi oscillation between bound impurity levels ${ }^{[12]}$ in doped GaAs. More recently, T. Kampfrath et al. performed the manipulation on the coherent spin waves in antiferromagnetic $\mathrm{NiO}$ by the magnetic component of intense Single-cycle terahertz pulses ${ }^{[13]}$.

The redistribution of electrons in momentum space in conduction band of semiconductors is expected to occur under the drive of the intense-field electromagnetic wave, which leads to the free-carriers nonlinear optics effects in the far-infrared frequency range. For instance, the intense THz electric field can lead to the ballistic transport of electrons in GaAs across half the Brillouin zone ${ }^{[14]}$. For the semiconductors materials with multi-valley conduction band such as GaAs, InGaAs, the electron at central energy valley ( $\Gamma$ valley) of conduction band can gain enough energy under high 
electric field to scatter into upper energy valleys (L, X valley). As a result, the electron mobility is decreased because the electron at $\mathrm{L}$ and $\mathrm{X}$ valley has larger effective mass than at $\Gamma$ valley. Recently, the intense few-cycle THz electric field pulse was utilized to reveal the ultrafast intervalley-electron-scattering dynamics in n-doped InGaAs and photoexcited $\mathrm{GaAs}^{[15,16]}$, which dominate the observed significant THz absorption bleaching. On the other hand, the electron velocity overshoot can take place over the sub-picosecond time scale under a strong step DC electric field due to the intervalley electron scattering in the semiconductor with multivalley conduction band structure. In this work, we employ the intense $\mathrm{THz}$ electric pulse with peak electric field higher than $170 \mathrm{kV} / \mathrm{cm}$ to access the nonlinear electron transport related to the $\Gamma$-L intervalley scattering in photoexcited $\mathrm{GaAs}$ and $\mathrm{Si}$. We demonstrate a method to explore the transient electron-velocity-overshoot phenomena via intense few-cycle $\mathrm{THz}$ electric pulse by using optical-pump - THz-probe (OPTP) experimental configuration.

\section{EXPERIMETAL}

The sample studied was $0.5 \mathrm{~mm}$-thickness, undoped-GaAs and Si single crystal wafer. In the OPTP experiment, the ALLS beam line ${ }^{[1]}$ used in the experiment provided $800 \mathrm{~nm}, 30 \mathrm{fs}$ laser pulses with energies as high as $38 \mathrm{~mJ}$ at a repetition rate of $100 \mathrm{~Hz}$. The beam was split into three portions, which are employed to produce $\mathrm{THz}$ pulse, excite optically sample and detect transmitted $\mathrm{THz}$ electric field, respectively. The few-cycle $\mathrm{THz}$ radiation with 0.1-3 THz frequency, $0.60 \mu \mathrm{J}$ energies, is generated by a large-aperture $\mathrm{ZnTe}$ optical rectification source. The collinear $\mathrm{THz}$ probe and $800 \mathrm{~nm}$ pump beam normally focusing on the sample without aperture have the spot diameter $1.6 \mathrm{~mm}$ and $12 \mathrm{~mm}$, respectively. The optical pump power on the sample is estimated lower than $0.9 \mathrm{~mW}$. The $\mathrm{THz}$ pulse electric field was detected by free-space electro-optic detection of a ZnTe crystal. The signal was monitored by the computer controlled lock-in amplifier. The THz transmissions with respect to the pump-probe delay were measured by controlling the mechanic delay lines. The experiments were done under a dry nitrogen purge at room temperature. In the measurement, the metal-wiregrid polarizers before and after sample are used to change the incident THz pulse energy and remove the saturation effect of ZnTe detector, respectively. The high-field THz pulse at focus position has the electric field amplitude of $170 \mathrm{kV} / \mathrm{cm}$ at main peak as estimated from the measured THz energy. For comparison, the low -field THz probe beam with peak electric field of $4 \mathrm{kV} / \mathrm{cm}$ is applied to detect the linear transmission.

\section{RESULTS AND DISCUSSION}

\subsection{THz absorption bleaching}

Figure 1 shows the temporal evolution of the electric-field-amplitude transmission of the $\mathrm{THz}$ main peak. Compared with the low-field $\mathrm{THz}$ probing, the time-dependent transmission probed by the high-field $\mathrm{THz}$ is increased pronouncedly for both $\mathrm{Si}$ and GaAs. The increment approaches to $32 \%$ after 2 ps of pump-probe delay for GaAs, when the carriers achieve the thermodynamic equilibrium. Figure 2 display the normalized $\mathrm{THz}$ waveforms measured at the pump-probe delay $\mathrm{t}=10 \mathrm{ps}$. The absorption bleaching can been seen clearly from the transmission of the overall waveforms, where the photoexcited carriers consume less $\mathrm{THz}$ electric field under high-field $\mathrm{THz}$ probing than low-field case. Especially, the tailing portion of high-field $\mathrm{THz}$ pulse travels through the photoexcited sample almost without electric-amplitude loss and phase shift. 

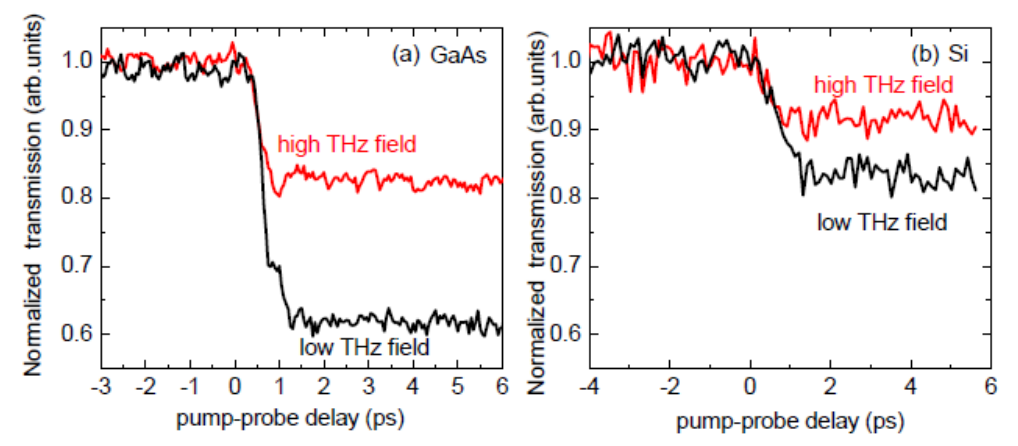

Figure 1: Normalized THz electric transmission of main peak as the function of the pump-probe time delay for GaAs (a) and Si (b), respectively.

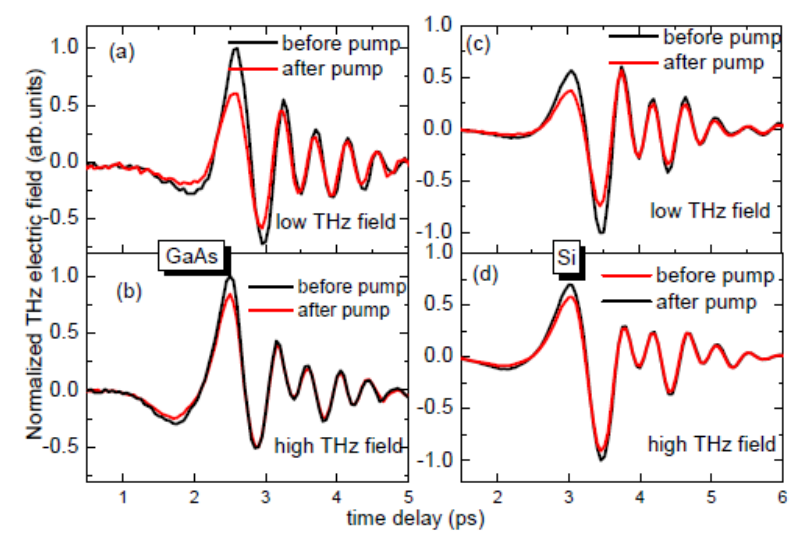

Figure 2: The normalized THz waveforms measured before and after pump ( $\mathrm{t}=10 \mathrm{ps}$ ) under low-field (a), (c) and high-field (b), (d)

$\mathrm{THz}$ probing for $\mathrm{GaAs}$ and $\mathrm{Si}$, respectively.

The $800 \mathrm{~nm}$-wavelength optical excitation injects the electrons into the $\Gamma$ valley and $\mathrm{X}$ valley of conduction band in GaAs and Si, respectively. The transmission depends on the conductivity of the free carriers in the thin conducting sheet [17]. Therefore, we propose that the absorption bleaching is attributed to the high-field electron transport. The electrons can gain far higher temperature than lattice under the applied strong electric field. As a consequence, the electrons in $\Gamma$ valley may be scattered into upper satellite valleys ( $\mathrm{L}, \mathrm{X}$ valleys) with larger effective mass (or lower mobility) when obtaining higher energy than the separation of adjacent two valleys. In addition, the electrons scattering rate would be increased when the polar optical phonon and intervalley scattering dominate ${ }^{[18]}$. On the other hand, since the optical phonon in GaAs has the lifetime (3-7 ps ${ }^{[19]}$ ) comparable to $\mathrm{THz}$ pulse length, the accumulation of emitted optical phonon from the electron scattering could take place under the strong $\mathrm{THz}$ field. The increase of the optical phonon population also decreases the electron mobility. As a result, the free-carrier conductivity is reduced due to the acceleration by the intense $\mathrm{THz}$ electric field, and therefore results in the increase of the $\mathrm{THz}$ transmission in photoexcited Si and GaAs. 


\subsection{Carrier velocity overshoot}

The THz electric field, $E_{\text {pump }}$, transmitted through the thin conducting photoexcited layer of conductivity $\sigma$ and thickness $d$ can be expressed in terms of the incident field, $E_{i}$, and current density $J$ as ${ }^{[20]}$

$$
E_{\text {pump }}=\frac{1}{Y_{0}+Y_{s}}\left(2 Y_{0} E_{i}-J d\right)
$$

where $Y_{0}=(377 \Omega)^{-1}$ and $Y_{s}=N Y_{0}$ are the free-space and sample admittances, respectively, and $N$ is the index of refraction of sample. The current density $J=q n v_{\Gamma}$ is determined by the carrier charge $q$, the carrier density $n$, and average drift velocity $v$. The transmitted THz electric field through the air-insulator interface before optical pump follows the equation,

$$
E_{\text {beforepump }}=\frac{1}{Y_{0}+Y_{s}}\left(2 Y_{0} E_{i}\right)
$$

Therefore, the carrier drift velocity is proportional to the transmitted $\mathrm{THz}$ electric field difference $(\delta E)$ between before and after optical pump, i.e., $v(t) \propto E_{\text {pump }}-E_{\text {beforepump }}$. In figure 3 , we present the normalized $\delta \mathrm{E}$ waveforms under low and high $\mathrm{THz}$ probe field for $\mathrm{Si}$ and GaAs, respectively. It can be seen that the high-field $\mathrm{THz}$ induced carrier average velocity exhibit remarkable saturation effect in the tailing portion of velocity waveform compared with low-field $\mathrm{THz}$ probe case. Especially, a pronouncedly drop occured to the carrier velocity drived by the high-field $\mathrm{THz}$ electric field in the case of photoexcited GaAs.

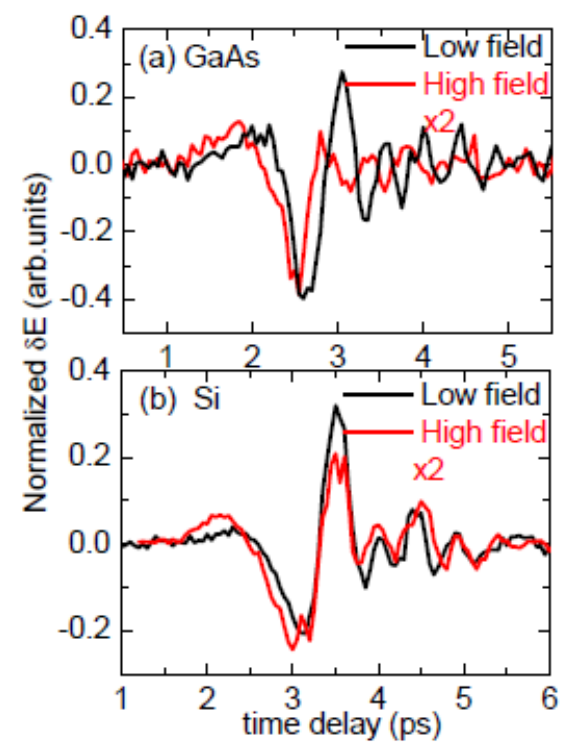

Figure 3: The experimental transmitted THz electric field difference between before and after optical pump, $\delta \mathrm{E}$, obtained from figure 2 for photoexcited GaAs (a) and Si (b), respectively. 
Under the strong DC electric field, the electron velocity tend to be saturated due to the energy exchange between electron and lattice via optical phonon scattering, the saturation velocity is described by the equation ${ }^{[21]}$

$$
v_{s}=\left(\frac{8}{3 \pi}\right)^{1 / 2}\left(\frac{\hbar \omega_{L O}}{m^{*}}\right)^{1 / 2}
$$

Where the $\hbar \omega_{L O}$ and $m^{*}$ denotes the optical phonon energy and electron effective mass, respectively. Since the time duration of electric field oscillation of the $\mathrm{THz}$ waveform is comparable to the electron scattering time, the strong $\mathrm{THz}$ transient can induce the electron velocity saturation in the manner of accelerating the electron by its electric field component.

Now we turn to the demonstration of extracting the electron average velocity in photoexcited GaAs. It should be noted that the contribution to the carrier mobility from photoexcited holes is large in the case Si. However, the carrier mobility due to hole is only $4 \%$ of electron mobility in photoexcited GaAs. Therefore, it is reasonable to neglect the nonlinear velocity from hole and consider the photoexcited GaAs as an electron conducting layer on the insulator substrate. The low-field THz complex conductivities in frequency domain for photoexcited GaAs follow the Drude mode as shown in the inset in figure 4 . The carrier scattering time, $\tau=170 \mathrm{fs}$, as well as the carrier density, $\mathrm{n}=1.7 \times 10^{17} \mathrm{~cm}^{-3}$, can be retrieved from the general Drude-conductivity fitting procedure, respectively ${ }^{[22,23]}$. Consequently, the average carrier velocity was obtained from the equation (1) and (2), and shown in figure 4 along with the incident $\mathrm{THz}$ electric field waveform. The carrier achieves its maximum velocity over $1 \times 10^{8} \mathrm{~cm} / \mathrm{s}$ by the acceleration of the two leading electric field oscillations followed by a drop to $-0.2 \times 10^{8} \mathrm{~cm} / \mathrm{s}$ corresponding to the third electric field oscillations in the $\mathrm{THz}$ waveform. The saturated electron velocity estimated from the equation (3) for GaAs is around $\mathrm{v}_{\mathrm{s}}=2 \times 10^{7} \mathrm{~cm} / \mathrm{s}$. Therefore, the extracted maximum and minimum carrier-velocity amplitude magnitude indicates that the electron velocity overshoot take place in the case of high-field THz probing for the phtoexcited GaAs. The electron velocity overshoot is triggered by the intense leading THz electric field oscillations, which accelerate electrons and push the electrons away Brillouin zone centre. Consequently, the electrons can overcome the $\Gamma$ - $\mathrm{L}$ valleys separation and be scattered into upper energy valleys with relatively large effective mass and small electron mobility. The dynamic modulation to the electron effective mass within sub-picosecond time scale leads to the nonlinear electron velocity overshoot transient. It should be mentioned that the electron velocity saturation due to the intavalley electron heating induced by intense $\mathrm{THz}$ electric field dominates in photoexcited Si. 


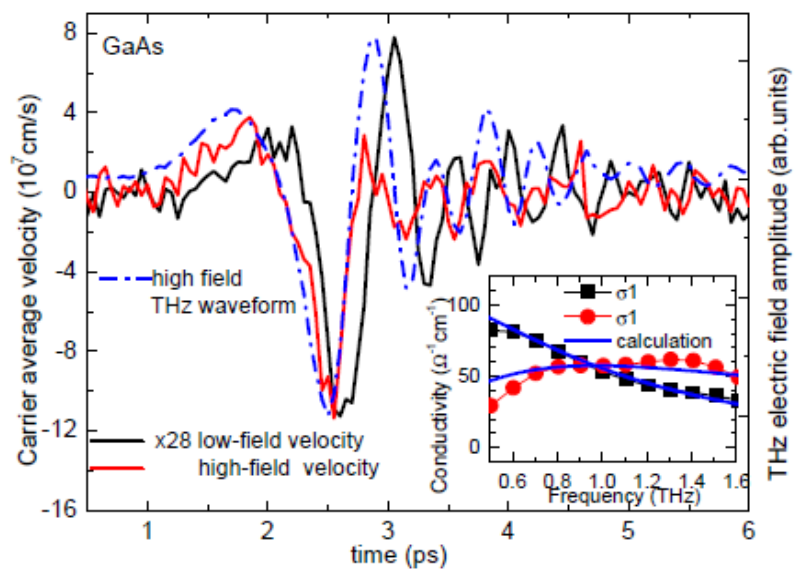

Figure 4: The carrier average velocity waveforms for photoexcited GaAs along with the incident THz electric field waveform (dash dot line). The inset presents the extracted complex conductivities as the function of frequency from the THz transmission. The $\sigma 1$ and $\sigma 1$ correspond to the real and imaginary part of conductivities, respectively. The blue solid lines denote the simulations to the experimental data by the Drude model.

\section{CONCLUSIONS}

We have observed nonlinear transient absorption bleaching of intense few-cycle terahertz (THz) pulses in photoexcited GaAs and Si by using optical-pump - THz-probe (OPTP) experimental configuration. The temporal waveforms of carrier average velocity were extracted from the transmitted $\mathrm{THz}$ electric field difference between before and after optical pump. A remarkable nonlinear carrier velocity saturation and overshoot in the carrier-average-velocity temporal profiles can be seen clearly in the case of high-electric-field $\mathrm{THz}$ probe. The observed $\mathrm{THz}$ dynamic absorption modulation and electron velocity overshoot is due to the THz-electric-field induced transient electron redistribution in conduction band.

\section{ACKNOWLEDGEMENT}

This work was support from NSERC, NSERC Strategic Projects and F. H. Su also wishes to acknowledge NSFC Grant Nos.11004199.

\section{REFERENCES}

[1] Blanchard, F., Razzari, L. , Bandulet, H.-C. , Sharma, G., Morandotti, R., Kieffer, J.-C ., Ozaki, T. , Reid, M. , Tiedje, H. F., Haugen, H. K., and Hegmann, F. A. "Generation of $1.5 \mu \mathrm{J}$ single-cycle terahertz pulses by optical rectification from a large aperture ZnTe crystal”, Opt. Express 15, 13212 (2007).

[2] Yeh, K.-L. , Hoffmann, M. C. , Hebling, J. , and Nelson, K. A. , "Generation of $10 \mu \mathrm{J}$ ultrashort terahertz pulses by optical rectification”, Appl. Phys. Lett. 90, 171121 (2007). 
[3] Ganichev, S. D. and Prettl, W. , [Intense Terahertz Excitation of Semiconductors ], Oxford University Press, Oxford (2006).

[4] Hebling, J., Yeh, K.-L. , Hoffmann, M. C., and Nelson, K. A., "High-power THz generation, THz nonlinear optics, and THz nonlinear spectroscopy”, IEEE J. Sel. Top. Quant. Electron. 14, 345 (2008).

[5] Nordstrom, K. B., Johnsen, K., Allen, S. J., Jauho, A.-P., Birnir, B. , Kono, J., Noda, T., Akiyama, H., and Sakaki, H., "Excitonic dynamical Franz-Keldysh effect”, Phys. Rev. Lett. 81, 457 (1998).

[6] Shinokita, K. , Hirori, H., Nagai, M., Satoh, N. , Kadoya, Y. , and Tanaka, K. , "Dynamical Franz-Keldysh effect in GaAs/AlGaAs multiple quantum wells induced by single-cycle terahertz pulses ", Appl. Phys. Lett. 97, 211902 (2010).

[7] Shen, Y., Watanabe, T. , Arena, D. A., Kao, C.-C. , Murphy, J. B., Tsang, T.Y., Wang, X. J. , and Carr, G. L. , "Nonlinear cross-phase modulation with intense single-cycle terahertz pulses", Phys. Rev. Lett. 99, 043901 (2007).

[8] Wen, H. , Wiczer, M., andLindenberg, A. M., "Ultrafast electron cascades in semiconductors driven by intense femtosecond terahertz pulses”, Phys. Rev. B 78, 125203 (2008).

[9]. Hoffmann, M. C., Hebling, J., Hwang, H. Y. , Yeh, K.-L., andNelson, K. A. , “Impact ionization in InSb probed by terahertz pump-terahertz probe spectroscopy”, Phys. Rev. B 79, 161201(R) (2009).

[10] Liu, J. , Kaur, G., and Zhang, X.-C., "Photoluminescence quenching dynamics in cadmium telluride and gallium arsenide induced by ultrashort terahertz pulse", Appl. Phys. Lett., 97, 111103 (2010).

[11] Gaal, P., Reimann, K. , Woerner, M. , Elsaesser, T., Hey, R., and Ploog, K. H. ,"Nonlinear terahertz response of n-type GaAs”, Phys. Rev. Lett. 96, 187402 (2006).

[12] Gaal, P. , Kuehn, W., Reimann, K., Woerner, M. , Elsaesser, T., Hey, R. , Lee, J. S. , and Schade, U. , “Carrier-wave Rabi flopping on radiatively coupled shallow donor transitions in $n$-type GaAs”, Phys. Rev. B 77, 235204 (2008).

[13] Kampfrath, T. , Sell, A., Klatt, G., Pashkin, A. , Mahrlein, S. , Dekorsy, T., Wolf, M., Fiebig, M. , Leitenstorfer, A. and Huber, R., "Coherent terahertz control of antiferromagnetic spin waves", nature photonics, 5, 31 (2011)

[14] Kuehn, W., Gaal, P. , Reimann, K. , Woerner, M. , Elsaesser, T. andHey, R., "Coherent Ballistic Motion of Electrons in a Periodic Potential", Phys. Rev. Lett. 104, 146602 (2010)

[15] Razzari, L., Su, F. H. , Sharma, G., Blanchard, F. , Ayesheshim, A., Bandulet, H. C. , Morandotti, R., Kieffer, J. C., Ozaki, T., Reid, M., and Hegmann, F. A., "Nonlinear ultrafast modulation of the optical absorption of intense few-cycle terahertz pulses in n -doped semiconductors," Phys. Rev. B, 79, 193204 (2009).

[16] Su, F. H. , Blanchard, F., Sharma, G., Razzari, L., Ayesheshim, A., Cocker, T. L., Titova, L. V., Ozaki, T., Kieffer, J. C. , Morandotti, R. , Reid, M., and Hegmann, F. A., "Terahertz pulse induced intervalley scattering in photoexcited GaAs," Opt. Express, 17, 9620 (2009).

[17] Nuss, M. C. and Orenstein, J. , [Terahertz time-domain spectroscopy, in Millimter and Submillimeter Wave Spectroscopy of Solids], Springer-Verlag, Berlin, Chap. 2., (1998).

[18] M. Lundstrom, Fundamentals of carrier transport, Cambridge university press chapter 7, page 282 (2000)

[19] Kash, J. A. , Hvam, J. M. , and Tsang, J. C. , "Subpicosecond Time-Resolved Raman Spectroscopy of LO Phonons in GaAs" Phys. Rev. Lett. 54, 2151 (1985)

[20] Nuss, M. C., Auston, D. H., andCapasso, F., "Direct subpicosecond measurement of carrier mobility of photoexcited electrons in gallium arsenide”, Phys. Rev. Lett. 58, 2355 (1987).

[21] Grundmann, M., [The physics of semiconductors], Springger, Chapter 8 (2006)

[22] Cooke, D. G. , Hegmann, F. A., Young, E. C. , andTiedje, T. , "Electron mobility in dilute GaAs bismide and nitride 
alloys measured by time-resolved terahertz spectroscopy”, Appl. Phys. Lett. 89, 122103 (2006).

[23] Beard, M. C., Turner, G. M. , and Schmuttenmaer, C. A. , "Transient photoconductivity in GaAs as measured by time-resolved terahertz spectroscopy”, Phys. Rev. B 62, 15764 (2000). 